\title{
Script processing in a natural situation
}

\author{
GLENN V. NAKAMURA \\ University of Illinois, Champaign, Illinois \\ ARTHUR C. GRAESSER and JUDY A. ZIMMERMAN \\ California State University, Fullerton, California \\ and \\ JAMES RIHA \\ Claremont Graduate School, Claremont, California
}

\begin{abstract}
The role of memory schemata in natural situations appears to be an important topic. An experiment is reported that addresses this topic by showing that a schema-based model developed under laboratory conditions is generalizable to a natural situation. Students in a laboratory section of a course received a lecture by a lecturer who performed actions that were relevant (e.g., underlining a word on the blackboard) or irrelevant (e.g., sipping coffee) with respect to a lecture script. A surprise recognition memory test showed that irrelevant actions were remembered better than relevant actions. This major result was consistent with a prediction made by a schema copy plus tag model.
\end{abstract}

Are memory phenomena that occur in the laboratory generalizable to various everyday life situations? To what degree can our memory theorizing be generalized from the laboratory to various everyday life situations? In the past, memory researchers have usually ignored these and other related questions. As a consequence, there is a need to know more about memory as it occurs in various natural situations (Neisser, 1976, 1978, 1982). The present study focuses on the generalizability of a schema-based model in explaining incidental recognition memory for actions performed by a lecturer in the course of giving a lecture.

During the last decade, the schema construct has provided an important and influential theoretical framework for guiding investigations of comprehension and memory (Alba \& Hasher, 1983; Anderson, 1977; Bower, Black, \& Turner, 1979; Bransford \& Johnson, 1972; Brewer \& Nakamura, 1984; Brewer \& Treyens, 1981; Friedman, 1979; Graesser, 1981; Graesser, Gordon, \& Sawyer, 1979; Graesser \& Nakamura, 1982; Kintsch \& van Dijk, 1978; Lichtenstein \& Brewer, 1980; Mandler, 1979; McDaniel, 1984; Minsky, 1975; Rubin \& Kontis, 1983; Rumelhart \& Ortony, 1977; Schank \& Abelson, 1977; Spiro, 1977; Thorndyke \& Hayes-Roth, 1979). In general, a schema can be conceptualized as an abstract generic knowledge structure that contains interrelationships among its typical components and the typical attributes of the components. A schema functions by guiding

This research was supported by National Institute of Mental Health Grant MH33491, awarded to Arthur Graesser. Authors Nakamura, Riha, and Zimmerman were members of Graesser's Cognitive Research Group when this study was conducted. We would like to thank Charles Clifton, Douglas Medin, and two anonymous reviewers for providing very helpful comments on an earlier draft of this manuscript. Requests for reprints should be addressed to Glenn Nakamura, Department of Psychology, University of Illinois, 603 E. Daniel, Champaign, IL 61820. our interpretations, inferences, expectations, attention, and later retrieval of various input.

A considerable amount of research activity has been devoted to scripts, which are one class of schemata (e.g., Abbott, Black, \& Smith, 1985; Bellezza \& Bower, 1982; Bower et al., 1979; Graesser et al., 1979; Graesser, Woll, Kolwalski, \& Smith, 1980; Haberlandt \& Bingham, 1984; Light \& Anderson, 1983; Owens, Bower, \& Black, 1979; Smith \& Graesser, 1981; Walker \& Yekovich, 1984). A script as a schematic knowledge structure represents the interrelationships among its components. The components refer to the roles, props, and actions that are found in various scripted activities, such as eating at a restaurant, washing a car, etc. As an example, consider a restaurant script that contains roles (e.g., hostess, waiter, customer, and cashier), props (e.g., tables, chairs, and menus), and an ordered series of actions (e.g., the customer reads the menu, the customer orders, the customer eats, and the customer pays the bill). The components or variables of the script have slots that can be filled by specific input that is encountered in a scripted activity. Alternatively, the variable slots of a script may be filled inferentially with typical values during comprehension. When a script's variable slots have been filled by explicit input and/or by inferences, the script is said to be instantiated. An instantiated script corresponds to a memory trace of a scripted activity that has been registered as having occurred at a specific time and place.

Much of the research on scripts has examined how script-based knowledge structures are utilized in the process of encoding, interpreting, drawing inferences, and guiding the retrieval of action sequences occurring in a script-based text. Usually, the actions presented in a scriptbased text vary in the degree to which they match the underlying variable slots of a script in memory. Some ac- 
tions in the text are irrelevant to the script. Irrelevant actions are those actions that do not occur frequently in a scripted activity, but that when they do occur they do not seem strange, bizarre, or inconsistent. Also, irrelevant actions are not instrumental in achieving the goals of the scripted activity. For example, putting a pen in one's pocket is irrelevant to the restaurant script, whereas walking on a table in a restaurant is bizarre, and not paying the bill after eating in a restaurant is inconsistent. Some actions in the text may be relevant to the script. Relevant actions are those actions that normally occur in a scripted activity and they are instrumental in achieving the goals of the scripted activity. For example, ordering dinner is relevant to the restaurant script. Thus, the actions presented in a text may vary in relevancy with respect to the underlying script in memory. One requirement of a script-based model is that it should be able to explain memory for relevant and irrelevant actions, since actions occurring in a scripted activity are rarely ever a perfect match to the variable slots of the underlying script.

A schema copy plus tag ( $\mathrm{SC}+\mathrm{T})$ model has been proposed and developed by Graesser (Graesser, 1981; Graesser et al., 1979; Graesser \& Nakamura, 1982; Graesser et al., 1980; Smith \& Graesser, 1981) in order to explain memory for relevant and irrelevant actions occurring in script-based passages. According to the SC $+\mathrm{T}$ model, a specific memory trace is constructed by the comprehender for every scripted activity that is read or heard. This memory trace contains a pointer to an instantiated script that has been copied from a permanent generic script. The instantiated script contains a subset of the script-relevant actions whose variable slots were activated by explicit information that was mentioned in the passage. In addition, there is a subset of script-relevant actions whose variable slots were activated by default. That is, the variable slots were activated as the result of scriptbased inferences occurring during comprehension of the text. Thus, the instantiated script contains activated explicit and inferred script-relevant actions. The memory trace also contains a set of tags, each of which corresponds to an irrelevant action. Each of these tags is a distinctive unit that is automatically incorporated into the memory trace.

The $\mathrm{SC}+\mathrm{T}$ model makes a number of predictions that have been confirmed by past research. However, only two of the more important predictions will be discussed here. First, the $\mathrm{SC}+\mathrm{T}$ model predicts that recognition memory discrimination should be better for irrelevant than for relevant actions. We define recognition memory discrimination for relevant and irrelevant actions as the ability to discriminate between target and distractor test actions. Specifically, for both relevant and irrelevant actions, the hit rate for target actions and the false-alarm rate for distractor actions is obtained in order to compute $\mathrm{D}^{\prime}$, which is a measure of memory discrimination. This first prediction follows from the representational assumptions of the $\mathrm{SC}+\mathrm{T}$ model. Explicitly stated irrelevant actions are separately and distinctively tagged in the memory trace.
In contrast, unstated irrelevant actions are not tagged in memory, since there is no script to infer them during comprehension. Thus, it should be easy to discriminate between those irrelevant actions that were stated and those that were not stated. It will be harder, however, to discriminate between those relevant actions that were stated and activated in the instantiated script and those relevant actions that were not stated, but were activated in the instantiated script due to script-based inferences occurring during comprehension. In addition, some of the unstated relevant actions may be inferred at the time of retrieval, and they are generated from the generic script. This memory discrimination prediction regarding relevant and irrelevant actions has been confirmed in previous studies (Graesser et al., 1980; Light \& Anderson, 1983; Smith \& Graesser, 1981). A second prediction is that there should be a higher false-alarm rate for relevant than for irrelevant actions. The rationale for this prediction is that the high false-alarm rate for relevant actions is the result of having stored comprehension-generated inferences in the instantiated script, as well as having generated inferences from the generic script at the time of retrieval. However, the false-alarm rate will be low for scriptirrelevant actions, because the instantiated script and the generic script do not guide inferencing for irrelevant actions. Research has supported this second prediction. A few studies have shown a high false-alarm rate for scriptrelevant actions, but a low false-alarm rate for scriptirrelevant actions (Graesser et al., 1979; Graesser et al., 1980; Light \& Anderson, 1983; Smith \& Graesser, 1981).

Previous script studies supporting the $\mathrm{SC}+\mathrm{T}$ model have shared two common attributes. First, the stimulus materials describing relevant and irrelevant actions were always restricted to prose passages. Second, the subjects always knew that they were in an experiment and therefore acquired the passages under intentional learning conditions.

In contrast, the present study was different from previous script studies in two major ways. First, in the present study, students in a laboratory section of a course heard a lecture given by a lecturer who performed a number of actions that varied in relevancy with respect to a lecture script. They were then given a surprise recognition memory test. Second, the students did not know that they were really in an experiment and therefore processed the relevant and irrelevant actions under incidental learning conditions. In this lecture situation, the $\mathrm{SC}+\mathrm{T}$ model predicted that irrelevant actions should show better recognition memory discrimination than would relevant actions. The model also predicted that the false-alarm rate would be higher for relevant than for irrelevant actions.

\section{METHOD}

\section{Subjects}

Forty undergraduates enrolled in an experimental psychology course at California State University, Fullerton, served as subjects. There were approximately 20 students in each of the two laboratory sections who saw the lecturer perform the actions. In addi- 
tion, 18 undergraduates provided normative ratings for the actions performed by the lecturer. They received course credit toward an introductory psychology course requirement.

\section{Materials}

There were two lectures, one for each laboratory section. The two lectures included a different sample of relevant and irrelevant actions. There were 5 relevant and 5 irrelevant actions performed in Lecture A, but not in Lecture B. Similarly, there were 5 relevant and 5 irrelevant actions performed in Lecture $B$, but not in Lecture A. Thus, there was a total of 20 actions, 10 of which were relevant and 10 of which were irrelevant to the lecture script.

A group of subjects provided normative ratings for the actions. This group rated each action by employing the following 6-point scale: $1=$ "the action is very atypical of a lecture," $2=$ "the action is moderately atypical of a lecture," $3=$ "uncertain, but the action is probably atypical of a lecture," $4=$ "uncertain, but the action is probably typical of a lecture," $5=$ "the action is moderately typical of a lecture," and $6=$ "the action is very typical of a lecture." Those actions that received mean typicality ratings greater than 4.14 and 4.07 were considered relevant in Lecture $A$ and Lecture B, respectively. Those actions that received mean typicality ratings less than 3.20 and 2.82 were considered irrelevant in Lecture A and Lecture B, respectively. Relevant actions in Lectures $A$ and $B$ had mean typicality ratings of $4.44(S D=.48)$ and $4.79(\mathrm{SD}=.64)$, respectively. Irrelevant actions in Lectures $A$ and $\mathrm{B}$ had mean typicality ratings of $2.61(\mathrm{SD}=.56)$ and 2.44 (SD $=.39$ ), respectively. The actions in Lecture A had mean typicality ratings that were closely matched to the mean typicality ratings for actions in Lecture B. The average absolute deviation was .38 between relevant actions in Lecture $A$ and Lecture $B$, whereas the average absolute deviation was .20 between irrelevant actions in Lecture $A$ and Lecture $B$.

Some examples of relevant actions were the lecturer's pointing to information on the blackboard, the lecturer's handing a student a sheet of paper, and the lecturer's opening and closing a book. Some examples of irrelevant actions were the lecturer's wiping off his glasses, the lecturer's bending a plastic coffee stirrer, and the lecturer's scratching his head. Table 1 shows a complete listing of all of the relevant and irrelevant actions that were performed in Lectures $\mathrm{A}$ and $\mathrm{B}$.

Table 1

Relevant and Irrelevant Actions Performed in Lectures A and B

\begin{tabular}{ll}
\hline \multicolumn{1}{c}{ Action } & Relevancy/Lecture \\
\hline Sitting on a corner of a table & Relevant-Lecture A \\
Pointing to information on the blackboard & Relevant-Lecture A \\
Opening and closing a book & Relevant-Lecture A \\
Moving an eraser to the blackboard & Relevant-Lecture A \\
Handing a student a sheet of paper & Relevant-Lecture A \\
Putting a piece of paper in a trash can & Irrelevant-Lecture A \\
Walking in and out of a side room & Irrelevant-Lecture A \\
Scratching head & Irrelevant-Lecture A \\
Wiping off glasses & Irrelevant-Lecture A \\
Bending a coffee stirrer & Irrelevant-Lecture A \\
Erasing a sentence off the blackboard & Relevant-Lecture B \\
Writing on lecture notes & Relevant-Lecture B \\
Leaning against the blackboard & Relevant-Lecture B \\
Getting a folder out of a briefcase & Relevant-Lecture B \\
Underlining a word on the blackboard & Relevant-Lecture B \\
Sipping coffee & Irrelevant-Lecture B \\
Taking off watch & Irrelevant-Lecture B \\
Dusting off pants & Irrelevant-Lecture B \\
Picking up a pencil off the floor & Irrelevant-Lecture B \\
Scratching knee & Irrelevant-Lecture B \\
\hline
\end{tabular}

Table 2

Recognition Memory Measures for Relevant and Irrelevant Actions

\begin{tabular}{lcc}
\hline Recognition Measure & Relevant Actions & Irrelevant Actions \\
\hline $\mathrm{D}^{\prime}$ Score & .15 & 1.02 \\
Hit Rate & .66 & .56 \\
False-Alarm Rate & .62 & .23 \\
\hline
\end{tabular}

\section{Procedure}

At the start of the lecture, the lecturer told the students that the lecture material was review and that they did not need to take any notes. The purpose of this comment was to encourage the students to look at the lecturer, rather than spend most of their time looking at their notebooks while taking notes. The lecturer then proceeded to deliver a 15 -min lecture. During the lecture, the lecturer performed a number of actions that varied in relevancy with respect to a lecture script. Both the relevant and irrelevant actions were performed in a smooth and nonobvious manner. After the lecture was over, there was a 20 -min intervening task. This task consisted of the lecturer's leading the students to a computer room, where he gave them a demonstration on how to operate a computer. While the students were in the computer room, a confederate went into the laboratory room in order to destroy any clues or evidence that could later be of some use in helping the students to reconstruct what actions had been performed. For example, for one of the lec tures, the confederate erased the blackboard so that the students would not be able to look at it in order to determine whether the lecturer had performed the action of underlining a word on the blackboard.

Following the computer demonstration, the students returned to the laboratory room and were immediately given an unexpected recognition memory test. The recognition memory test contained the 10 Lecture $A$ actions ( 5 relevant and 5 irrelevant actions) and the 10 Lecture $B$ actions ( 5 relevant and 5 irrelevant actions). The lecturer told the students that some of the actions on the recognition test were performed during the lecture, whereas some were not performed. The students were instructed to rate each test action according to a 6-point recognition scale: $1=$ "definitely certain that the action was not performed by the lecturer," $2=$ "fairly certain that the action was not performed by the lecturer," $3=$ "uncertain, but think that the action was not performed by the lecturer," $4=$ "uncertain, but think that the action was performed by the lecturer," $5=$ "fairly certain that the action was performed by the lecturer," and $6=$ "definitely certain that the action was performed by the lecturer."

\section{RESULTS}

Table 2 shows the mean $\mathrm{D}^{\prime}$ scores, the mean hit rates (proportion of 4,5 , and 6 ratings for presented actions), and the mean false-alarm rates (proportion of 4,5 , and 6 ratings for unpresented actions) for relevant and irrelevant actions. Table 2 shows an important finding, which is that memory discrimination, as measured by $\mathrm{D}^{\prime}$, was better for irrelevant than for relevant actions $[F(1,39)=$ $21.00, \mathrm{p}<.01, \mathrm{MSe}=.71]$. This finding supported a prediction of the $\mathrm{SC}+\mathrm{T}$ model. A further inspection of Table 2 shows that the better memory discrimination for irrelevant than for relevant actions is due primarily to the fact that the false-alarm rate was low for irrelevant actions, but high for relevant actions. The false-alarm rate for relevant actions is almost three times larger than the false-alarm rate for irrelevant actions $[F(1,39)=82.50$, $\mathrm{p}<.01, \mathrm{MSe}=.03]$. This pattern of false-alarm rates supported another prediction of the SC $+\mathrm{T}$ model. Finally, Table 2 shows that the hit rate is slightly higher 
for relevant than for irrelevant actions $[\mathrm{F}(1,39)=5.77$, $\mathrm{p}<.03, \mathrm{MSe}=.04]$.

\section{DISCUSSION}

Recognition memory discrimination was better for irrelevant than for relevant actions. In addition, the falsealarm rate was higher for relevant than for irrelevant actions. These findings provide evidence that the $\mathrm{SC}+\mathrm{T}$ model can be generalized to account for memory performance in a natural situation. In the past, the $\mathrm{SC}+\mathrm{T}$ model has been shown to account for patterns of memory performance when the actions were presented in script-based passages and under intentional learning conditions (Graesser et al., 1979; Graesser et al., 1980; Light \& Anderson, 1983; Smith \& Graesser, 1981). The SC+T model claims that irrelevant actions will show good memory discrimination, because it is easy to discriminate between stated irrelevant actions that are tagged in memory and unstated irrelevant actions that are not tagged in memory. The SC+T model claims that relevant actions will show poorer memory discrimination, because it is difficult to discriminate between those actions that were explicitly stated and those actions that were unstated but inferred.

The results of this present experiment are encouraging in that they appear to contribute to our understanding of memory as it occurs under natural circumstances. In addition, the results of the present study provide a link to several other studies addressing memory for information encountered in a natural situation. One study by Kintsch and Bates (1977) investigated incidental recognition memory for relevant and irrelevant statements made by a lecturer. The irrelevant statements corresponded to jokes, announcements, etc., whereas the relevant statements corresponded to lecture material on either the history of intelligence testing or Freudian theory. The results showed that recognition memory was better for irrelevant than for relevant statements. Recognition memory in the Kintsch and Bates study was defined as the recognition ratings given to old and new test statements. The better memory for irrelevant statements was due primarily to the correct rejections of new test statements, rather than to the correct recognition of old test statements. Along similar lines, in the present study, the better memory for irrelevant actions was due primarily to a low false-alarm rate, rather than to a high hit rate.

In addition, Brewer and Treyens (1981) investigated the role of a room schema on incidental memory for objects in a graduate student's office. The office contained a number of objects varying in schema-expectancy. The schemaexpected objects were those objects that were likely to appear in the room (e.g., a desk, a chair, etc.). In contrast, schema-unexpected objects were those objects that were not likely to appear in the room (e.g., tennis racket, cactus plant, etc.). Recognition memory in the Brewer and Treyens study was defined as the recognition ratings given to both old and new objects. Recognition ratings were positively correlated with the schema-expectancy ratings for both old and new objects. The positive correlation between recognition ratings and schema-expectancy ratings for old objects indicated that schema-expected objects were more likely to be correctly recognized than schema-unexpected objects. This finding is similar to one of our findings, which was that the hit rate was slightly higher for script-relevant than for script-irrelevant actions. Also, the positive correlation between recognition ratings and schema-expectancy ratings for new objects indicated that schema-expected objects were more likely to be falsely recognized than were schema-unexpected objects. The high false-alarm rate for schema-expected objects supported an interpretation that claimed that the room schema was involved in inferencing. This finding of a high falsealarm rate for schema-expected objects and its interpretation is parallel to our account for why script-relevant actions had a high false-alarm rate.

Research conducted within different natural situations appears to be important, because such a research strategy may allow researchers to uncover phenomena corresponding to different knowledge domains (Brewer \& Nakamura, 1984). In addition, within a given natural situation, there may be different knowledge domains that are naturally accessed and used. For example, there is a room schema that corresponds to objects found in a lecture room. There is a script schema that corresponds to actions performed by the lecturer. Finally, there are schemata that correspond to the lecture material. Memory in natural situations involves many different kinds of schemata.

\section{REFERENCES}

Abbott, V., Black, J. B., \& Smith, E. E. (1985). The representation of scripts in memory. Journal of Memory and Language, 24, 179-199

Alba, J. W., \& Hasher, L. (1983). Is memory schematic? Psychological Bulletin, 93, 203-231.

ANDERSON, R. C. (1977). The notion of schemata and the educational enterprise: General discussion of the conference. In R. C. Anderson, R. J. Spiro, \& W. E. Montague (Eds.), Schooling and the acquisition of knowledge. Hillsdale, NJ: Erlbaum.

BellezZA, F. S., \& Bower, G. H. (1982). Remembering script-based text. Poetics, 11, 1-23.

Bower, G. H., Black, J. B., \& Turner, T. J. (1979). Scripts in comprehension and memory. Cognitive Psychology, 11, 177-220.

BRANSFord, J. D., \& Johnson, M. K. (1972). Contextual prerequisites for understanding: Some investigations of comprehension and recall. Joumal of Verbal Learning and Verbal Behavior, 11, 717-726.

Brewer, W. F., \& Nakamura, G. V. (1984). The nature and functions of schemas. In R. S. Wyer \& T. K. Srull (Eds.), Handbook of social cognition. Hillsdale, NJ: Erlbaum.

Brewer, W. F., \& TREYeNS, J. C. (1981). Role of schemata in memory for places. Cognitive Psychology, 13, 207-230.

Friedman, A. (1979). Framing pictures: The role of knowledge in automatized encoding for gist. Journal of Experimental Psychology: General, 108, 316-355.

Graesser, A. C. (1981). Prose comprehension beyond the word. New York: Springer.

Graesser, A. C., Gordon, S. E., SAwyer, J. D. (1979). Recognition memory for typical and atypical actions in scripted activities: Tests of a script pointer + tag hypothesis. Journal of Verbal Learning and Verbal Behavior, 18, 319-332. 
Graesser, A. C., \& Nakamura, G. V. (1982). The impact of a schema on comprehension and memory. In G. H. Bower (Ed.), The psychology of learning and motivation: Advances in research and theory. New York: Academic Press.

Graesser, A. C., Woll, S. B., Kolwalski, D. J., \& SMith, D. A. (1980). Memory for typical and atypical actions in scripted activities. Journal of Experimental Psychology: Human Learning and Memory, 6, 503-515.

HaberlandT, K., \& Bingham, G. (1984). The effect of input direction on the processing of script statements. Journal of Verbal Learning and Verbal Behavior, 23, 162-177.

KINTSCH, W., \& BATES, E. (1977). Recognition memory for statements from a classroom lecture. Journal of Experimental Psychology: $\mathrm{Hu}$ man Learning and Memory, 3, 150-159.

KINTSCH, W., \& VAN DiJK, T. A. (1978). Toward a model of text comprehension and production. Psychological Review, 85, 363-394.

Lichtenstein, E. H., \& BreWER, W. F. (1980). Memory for goaldirected events. Cognitive Psychology, 12, 412-445.

Light, L. L., \& ANDERSON, P. A. (1983). Memory for scripts in young and older adults. Memory \& Cognition, 1, 435-444.

MandleR, J. M. (1979). Categorical and schematic organization in memory, In C. R. Puff (Ed.), Memory organization and structure. New York: Academic Press.

MCDaniel, M. A. (1984). The role of elaborative processes and schema processes in story memory. Memory \& Cognition, 12, 46-51.

MiNSKY, M. (1975). A framework for representing knowledge. In P. H. Winston (Ed.), The psychology of computer vision. New York: McGraw-Hill.

NeISSER, U. (1976). Cognition and reality. San Francisco: Freeman. NeIsser, U. (1978). Memory: What are the important questions? In
M. M. Gruneberg, P. E. Morris, \& R. N. Sykes (Eds.), Practical aspects of memory. London: Academic Press.

NeISSER, U. (1982). Memory observed. San Francisco: Freeman.

Owens, J., Bower, G. H., \& BlACK, J. B. (1979). The 'soap opera' effect in story recall. Memory \& Cognition, 7, 185-191.

Rubin, D. C., \& Kontis, T. C. (1983). A schema for common cents. Memory \& Cognition, 11, 335-341.

Rumelhart, D. E., \& ORTony, A. (1977). The representation of knowledge in memory. In R. C. Anderson, R. J. Spiro, \& W. E. Montague (Eds.), Schooling and the acquisition of knowledge. Hillsdale, NJ: Erlbaum.

SChANK, R. C., \& ABELson, R. (1977). Scripts, plans, goals, and understanding. Hillsdale, NJ: Erlbaum.

SMITH, D. A., \& Graesser, A. C. (1981). Memory for actions in scripted activities as a function of typicality, retention interval, and retrieval task. Memory \& Cognition, 9, 550-559.

SpIRo, R. J. (1977). Remembering information from text: The 'state of the schema' approach. In R. C. Anderson, R. J. Spiro, \& W. E. Montague (Eds.), Schooling and the acquisition of knowledge. Hillsdale, NJ: Erlbaum.

ThORNDYKe, P. W., \& HAYes-Roth, B. (1979). The use of schemata in the acquisition and transfer of knowledge. Cognitive Psychology, 11, 82-106.

Walker, C. H., \& Yekovich, F. R. (1984). Script-based inferences: Effects of text and knowledge variables on recognition memory. Journal of Verbal Learning and Verbal Behavior, 23, 357-370.

(Manuscript received July 23, 1984; revision accepted for publication January 11, 1985.) 